\title{
ON A DIOPHANTINE PROBLEM
}

\author{
J. B. ROBERTS
}

Introduction. If $a_{1}, a_{2}, \ldots, a_{k}$ are relatively prime positive integers then the equation

$$
a_{1} x_{1}+\ldots+a_{k} x_{k}=n
$$

always has solutions in non-negative $x_{i}$ for $n$ sufficiently large. Sylvester called the number of non-negative solutions of (1) the denumerant of the equation. We shall denote the denumerant by $n\left(a_{1}, \ldots, a_{k}\right)$.

We define $N_{j}\left(a_{1}, \ldots, a_{k}\right)$ to be the smallest positive integer such that $n\left(a_{1}, \ldots, a_{k}\right) \geqslant j$ for all $n \geqslant N_{j}\left(a_{1}, \ldots, a_{k}\right)$.

Using the known result

$$
n(1,2,3)=\left[\left(n^{2}+6 n+15\right) / 12\right]
$$

we can readily show that

$$
N_{j}(1,2,3)=[\sqrt{ }(12 j-6)-2] .
$$

In general the computation of $N_{j}\left(a_{1}, \ldots, a_{k}\right)$ is a difficult job. Our interest here is in $N_{1}\left(a_{1}, \ldots, a_{k}\right)$. The present author has given (4) the value of $N_{1}\left(a_{1}, \ldots, a_{k}\right)$ when the $a_{i}$ are in arithmetic progression. Brauer and Seelbinder $(2 ; 3)$ have given various upper bounds for $N_{1}\left(a_{1}, \ldots, a_{k}\right)$ in the general case. In this paper we concern ourselves with an upper bound for $N_{1}(m, m+a, m+b)$ where $(a, b)=1$. Our upper bound is in many cases best possible. We also give a theorem concerning an upper bound for

$$
N_{1}\left(m, m+a_{1}, \ldots, m+a_{k}\right)
$$

where $a_{1}, a_{2}, \ldots, a_{k}$ are relatively prime positive integers.

1. Three lemmas. Throughout this section

$$
0<a<b,(a, b)=1, P=(a-1)(b-1) .
$$

Since $N_{1}(a, b)=P(\mathbf{1}$, p. 124$)$, the equation $a x+b y=n$ is solvable in nonnegative integers for all $n \geqslant P$. The non-negative solution with smallest $x$ is denoted by $x_{n}, y_{n}$. If $x, y$ is any non-negative solution of $a x+b y=n$ then

$$
x+y \geqslant x_{n}+y_{n} \text {. }
$$

Also

$$
x_{n+b}+y_{n+b}=x_{n}+y_{n}+1 \text {. }
$$

Received April 29, 1956. 
Since $(a, b)=1$, the numbers $\alpha a$ for $0 \leqslant \alpha \leqslant b-1$ constitute a complete system of residues modulo $b$. Hence $n \equiv \alpha a(\bmod b)$ has a unique solution with $0 \leqslant \alpha \leqslant b-1$. Denote this solution by $\alpha_{n}$.

LEMMA 1. $x_{n}=\alpha_{n}, \quad y_{n}=\left(n-a \alpha_{n}\right) / b$.

Proof. Clearly

$$
a \alpha_{n}+b\left(\left(n-a \alpha_{n}\right) / b\right)=n .
$$

Hence all solutions of $a x+b y=n$ are given by

$$
\alpha_{n}+b t,\left(n-a \alpha_{n}\right) / b-a t .
$$

For non-negative solutions we must have

$$
t \geqslant-\alpha_{n} / b>-1
$$

Hence all non-negative solutions have $x \geqslant \alpha_{n}$.

Lemma 2. If $n>P, \alpha_{n}=b-1$ and $1 \leqslant c \leqslant n-P$ then

$$
x_{n}+y_{n} \geqslant x_{n-c}+y_{n-c}+1 \text {. }
$$

Proof. We have

$$
\begin{aligned}
x_{n}+y_{n} & =\alpha_{n}+\left(n-a \alpha_{n}\right) / b=\left(n+\alpha_{n}(b-a)\right) / b \\
& =(n+(b-1)(b-a)) / b>\left(n-c+\alpha_{n-c}(b-a)\right) / b \\
& =x_{n-c}+y_{n-c} .
\end{aligned}
$$

LEMMA 3.

$$
\max _{n \in K}\left(x_{n}+y_{n}\right) \leqslant b-2+[m / b]
$$

where $K$ consists of those $n$ satisfying $P \leqslant n \leqslant P+m-1, m \geqslant 1$.

Proof. Let $B$ be the smallest integer greater than $P+m-1$ which has $\alpha_{B}=b-1$. Then

$$
B>P+m-1 \geqslant n
$$

and therefore $B-n \geqslant 1$. Also $B-n \leqslant B-P$. Hence taking the $n, c$ of Lemma 2 to be $B, B-n$ respectively, we see that

$$
x_{B}+y_{B} \geqslant x_{B-(B-n)}+y_{B-(B-n)}+1=x_{n}+y_{n}+1 .
$$

This is true for all $n \in K$, so

$$
\max _{n \in K}\left(x_{n}+y_{n}\right) \leqslant x_{B}+y_{B}-1=\left(B+\alpha_{B}(b-a)\right) / b-1 .
$$

For arbitrary $m$ we have

$$
B=P+[m / b] b-1+b .
$$

Hence

$$
\begin{aligned}
\max _{n \in K}\left(x_{n}+y_{n}\right) \leqslant(P+ & {[m / b] b-1+b+(b-1)(b-a)) / b-1 } \\
& =b-2+[m / b] .
\end{aligned}
$$




\section{The main theorem.}

Theorem 1. $N_{1}(m, m+a, m+b) \leqslant m(b-2+[m / b])+(a-1)(b-1)$ where $0<a<b,(a, b)=1, m \geqslant 2$.

Proof. Let $P, K, x_{n}, y_{n}$ be as in $\$ 1$. Define

$$
Q=\max _{n \in K}\left(x_{n}+y_{n}\right) \text {. }
$$

Now define $\bar{x}_{j}, \bar{y}_{j}, \bar{z}_{j}$ for $j \geqslant P$ by the following :

$$
\begin{aligned}
& \bar{x}_{j}=x_{i}, \bar{y}_{j}=y_{i} \text { for } j \equiv i(\bmod m), \quad P \leqslant i \leqslant P+m-1, \\
& \bar{z}_{j}=Q+[(j-P) / m]-\bar{x}_{j}-\bar{y}_{j} .
\end{aligned}
$$

Then

$$
\begin{aligned}
m \bar{z}_{j}+(m+a) \bar{x}_{j}+(m+b) \bar{y}_{j} & =m\left(\bar{z}_{j}+\bar{x}_{j}+\bar{y}_{j}\right)+a \bar{x}_{j}+b \bar{y}_{j} \\
& =m Q+m[(j-P) / m]+a \bar{x}_{j}+b \bar{y}_{j} .
\end{aligned}
$$

As $j$ runs over

$$
P+s m, P+s m+1, \ldots, P+s m+m-1
$$

for $s \geqslant 0$, the right side of this equation runs over

$$
m Q+m s+P, m Q+m s+P+1, \ldots, m Q+m s+P+m-1 .
$$

Hence as $j$ runs over the integers greater than or equal to $P$ and $s$ runs over the integers greater than or equal to 0 , the left side of $(2)$ runs over the integers greater than or equal to $P+m Q$. Replacing $Q$ by its upper bound from Lemma 3 now gives the desired result.

Essentially the same proof yields the following:

Theorem 2. $N_{1}\left(m, m+a_{1}, \ldots, m+a_{k}\right) \leqslant P+m Q$ where $a_{1}, a_{2}, \ldots, a_{k}$ are relatively prime positive integers,

$$
P=N_{1}\left(a_{1}, \ldots, a_{k}\right), \quad Q=\max _{n \in K}\left(x_{1 n}+\ldots+x_{k n}\right),
$$

$K$ is the set of $n$ such that $P \leqslant n \leqslant P+m-1$, and $x_{1 n}, \ldots, x_{k n}$ is a nonnegative solution of (1) with smallest sum.

3. Special cases. Let $\widetilde{N}_{1}(m, m+a, m+b)$ denote the upper bound in Theorem 1 . We compare $\widetilde{N}_{1}$ with $N_{1}$ in a few special cases.

(a) By the main result in (4; see also 2, Theorem 7, p. 310),

$$
N_{1}(m, m+1, m+2)=m\left[\frac{1}{2} m\right]=\tilde{N}_{1}(m, m+1, m+2) .
$$

(b) By a result stated in (4),

$$
N_{1}(m, m+1, m+b)=\widetilde{N}_{1}(m, m+1, m+b)
$$

for

$$
m \equiv-1(\bmod b), \quad m \geqslant b^{2}-5 b+3 ;
$$


and

$$
N_{1}(m, m+1, m+b)=\widetilde{N}_{1}(m, m+1, m+b)-(m-b[m / b])
$$

for

$$
m \equiv-1(\bmod b), \quad m \geqslant b^{2}-4 b+2 .
$$

(c) By direct evaluation it is not difficult to show

$$
N_{1}(m, m+2, m+3)=\widetilde{N}_{1}(m, m+2, m+3) .
$$

(d) By computation for $2 \leqslant m \leqslant 16$ we find

$$
N_{1}(m, m+2, m+5)=\widetilde{N}_{1}(m, m+2, m+5)
$$

for

$$
m=5,8,10,13,14,15
$$

and not for

$$
m=2,3,4,6,7,9,11,12,16 .
$$

\section{REFERENCES}

1. P. Bachmann, Niedere Zahlentheorie, 2 (Leipzig, 1910).

2. A. Brauer, On a problem in partitions, Amer. J. Math., 64 (1942), 299-312.

3. A. Brauer and B. M. Seelbinder, On a problem in partitions II, Amer. J. Math., 76 (1954), 343-346.

4. J. B. Roberts, Note on linear forms, Proc. Amer. Math. Soc., 7 (1956), 465-469.

Wesleyan University

Middletown, Conn. 